\title{
Diet breadth, coexistence and rarity in bumblebees
}

Dave Goulson, Gillian C. Lye and Ben Darvill

School of Biological \& Environmental Sciences, University of Stirling, Stirling, UK Tel: +44-786-467759

Email: Dave.Goulson@stir.ac.uk

Key Words: Hymenoptera; Bombus; community composition; forage use; tongue length; pollen; competition 


\section{ABSTRACT}

Factors that determine the relative abundance of bumblebee species remain poorly understood, rendering management of rare and declining species difficult. Studies of bumblebee communities in the Americas suggest that there are strong competitive interactions between species with similar length tongues, and that this competition determines the relative abundance of species. In contrast, in Europe it is common to observe several short-tongued species coexisting with little or no evidence for competition shaping community structure. In this study we examine patterns of abundance and distribution in one of the most diverse bumblebee communities in Europe, found in the mountains of southern Poland. We quantify forage use when collecting nectar and pollen for 23 bumblebee species, and examine patterns of co-occurrence and niche overlap to determine whether there is evidence for inter-specific competition. We also test whether rarity can be explained by diet breadth. Up to 16 species were found coexisting within single sites, with species richness peaking in mountain pasture at $\sim 1000 \mathrm{~m}$ altitude. Results concur with previous studies indicating that the majority of pollen collected by bumblebees is from Fabaceae, but that some bee species (e.g. B. ruderatus) are much more heavily dependent on Fabaceae than others (e.g. B. lucorum). Those species that forage primarily on Fabaceae tended to have long tongues. In common with studies in the UK, diet breadth was correlated with abundance: rarer species tended to visit fewer flower species, after correcting for differences in sample size. No evidence was found for similarity in tongue length or dietary overlap influencing the likelihood of co-occurrence of species. However, the most abundant species (which co-occurred at most sites) occupied distinct dietary niche space. While species with tongues of similar length tended, overall, to have higher dietary niche overlap, among the group of abundant short-tongued species that commonly co-occurred there was marked dietary differentiation which may explain their coexistence. 


\section{INTRODUCTION}

Bumblebees (Bombus spp.) and other wild pollinators are widely perceived to have suffered declines in abundance and range contractions in the last 70 years across much of western Europe and North America (Williams 1982, 1986, 2005, Rasmont 1995, Kosior 1995, Buchmann and Nabhan 1996, Westrich 1996, Westrich et al. 1998, Goulson 2003a,b, Kosior et al. 2007). The consensus is that declines in numbers of bumblebees have been driven by intensification of farming practices leading to loss of wildflowers and nesting sites (Williams 1986, Osborne and Corbet 1994, Goulson 2003a). Interestingly, a small number of bumblebee species seem to have coped well with agricultural changes, and remain widespread and abundant even in intensively farmed areas. Understanding why some species remain common while others are in rapid decline is key to developing appropriate conservation strategies for the threatened species. A number of studies have attempted to explain patterns of abundance and community composition in bumblebees.

In the 1970's and 1980's studies of competition were much in vogue, and bumblebees attracted attention because several species can commonly be found coexisting despite apparently very similar niches: all bumblebees are hairy, endothermic and are large relative to most other bees. They exhibit little morphological variation other than in size; most have an annual cycle and are active at similar times of the year; and they all feed almost exclusively on nectar and pollen throughout their lives. One might expect fierce interspecific competition to shape bumblebee communities (Brian 1954, Heinrich 1976).

One niche dimension in which bumblebee species have long been known to vary is tongue length, which leads to differences in floral preferences between species (Brian 1957, 
Hobbs et al. 1961, Hobbs 1962, Holm 1966, Ranta and Lundberg 1980, Harder 1985) and resource partitioning with respect to tongue length has long been thought to be an important factor in allowing a number of bumblebee species with otherwise very similar biology to coexist (Heinrich 1976a, Teräs 1976, Inouye 1978, 1980, Barrow and Pickard 1984, Harder 1985, Johnson 1986, Graham and Jones 1996). However, it must be noted that bumblebees exhibit great size (and hence tongue length) variation within species, which increases resource overlap between species but allows dietary specialization among nest mates (Goulson et al. 2002a, Peat et al. 2005a, b)

Studies in Colorado appear to suggest that species with similar length tongues exclude one another from floral resources (Inouye 1978), and that at any particular site only four species could coexist, one long, one medium and one short tongued species plus a fourth short tongued species that was also a nectar robber (Pyke 1982). More recent studies in Europe have failed to find such clear patterns. North and Central European bumblebee communities commonly consist of six to eleven species, with considerable overlap in tongue lengths (Ranta et al. 1981, Ranta and Vepsäläinen 1981). Several short-tongued species are commonly found to coexist. In the UK, six bumblebee species are abundant, widespread, and generally occur together. Yet four of them have short tongues of very similar length (Williams 1989, Goulson et al. 1998, Kells et al. 2001). Studies of local assemblages of bumblebees have failed to find any pattern in the tongue lengths of species in relation to their co-occurrence (Ranta 1982, 1983, Ranta and Tiainen 1982, Williams 1985b, 1988). Ranta and Vepsäläinen (1981) attribute coexistence of species with similar tongue lengths in Europe to spatio-temporal heterogeneity in nest distribution and floral resources (see also Tepedino and Stanton 1981). They argue that the strength and direction of competitive interactions between colonies of different species will fluctuate greatly over the season (as the availability of different flower species varies) and also from 
nest to nest, since flower distributions are patchy. Thus competition will not drive species to local extinction.

More recently, concerns over the dramatic declines of some bumblebee species have led research to focus on trying to understand why some species appear to be both rarer and more susceptible to environmental change than others. Based on studies of forage use by UK bumblebee species, Goulson et al. $(2005,2006)$ argue that the rare species tend to be long tongued and have narrower diets, with a very large proportion of the pollen they collect being from Fabaceae. These species are associated with Fabaceae-rich unimproved grasslands, a habitat which has been largely eradicated in western Europe. In contrast the common species tend to have broad foraging preferences and readily encompass non-native garden plants and mass-flowering crops in their diets (see also Goulson et al. 2002b). Williams (2005) showed that rare and declining species in Britain tend to have small geographic ranges within Europe. In a more detailed comparison of two rapidly declining species, $B$. distinguendus and $B$. sylvarum, with the ubiquitous $B$. pascuorum, Williams et al. (2007) show that the two declining species had narrower climatic niche space across Europe prior to their major declines, and that they have contracted their range in the UK away from the margins of their climatic tolerance. In contrast, Fitzpatrick et al. (2007) examined the Irish bee fauna (which consists of a subset of the UK species) and found that neither climatic range nor diet breadth correlated with decline. The only factor to significantly correlate with decline was queen emergence time, with later emerging species having disappeared from more former locations. It is clear from the discrepancies between these studies that we do not as yet have a full understanding of the factors affecting the relative abundance and susceptibility to habitat loss of bumblebee species, even in the intensively-studied British and Irish fauna. 
Here we examine community composition, diet breadth and abundance in one of the richest bumblebee communities remaining in Europe, in the mountains of southern Poland. The area is characterized by extensive agricultural systems and little mechanization. Horses remain the primary source of power on farms, crops are still cut mainly by hand, and field sizes are typically $<1 \mathrm{Ha}$. This farming system is probably similar to that found in much of western Europe 100 years ago, and as a result, some bumblebee species that are now exceedingly scarce in western Europe remain abundant in Poland. We test whether the association between rarity and diet breadth described by Goulson et al. (2005) for UK bumblebees is found in this more diverse bee community, and we examine whether community composition is shaped by competition i.e. can species with similar tongue length and high dietary overlap coexist?

\section{METHODS}

Bumblebee forage use was quantified using the same methodology at 32 sites in the Gorce and Tatra mountains of southern Poland (Appendix 1). Study sites were chosen to provide abundant bumblebee forage and to span a range of altitudes. Data were collected between 31 July and 12 August 2006, at the peak of the colony cycle for most species. The area is predominantly agricultural, with small fields (most $<1 \mathrm{Ha}$ ) and little mechanisation. Farms are mixed arable and livestock up to about $900 \mathrm{~m}$, above which the predominant land use is montane pasture. Only broadly similar habitats were included i.e. open flower-rich pastures and field margins.

Each site consisted of a circle of approximately $100 \mathrm{~m}$ radius, and was searched for one man hour, precisely following methods previously used by Goulson and Darvill (2004), Goulson and Hanley (2004) and Goulson et al. (2005), to enable comparisons between the data sets. All searches were conducted between $0800 \mathrm{~h}$ and $1700 \mathrm{~h}$, and during warm dry 
weather favourable to bee activity. All Bombus species were recorded, but not other bee species. Species which are difficult to distinguish on the wing were captured and examined with a hand lens. The area was searched systematically to avoid recording the same bees more than once, but this probably occasionally occurred. The flower species that the bee visited was recorded, and the bee was briefly observed to determine whether it was collecting pollen or nectar. Bees were classified as nectar collectors (those not collecting pollen) or pollen collectors (those observed actively grooming pollen into their corbiculae), but it must be noted that most pollen collectors were also collecting nectar. The total number of inflorescences within the search area (the circle of $100 \mathrm{~m}$ radius) was estimated for each forage species present. The proportion of visits by bees of each species (all castes combined) to each plant family was examined using principal components analysis in SPSS 11.0, with separate analyses for pollen-collecting and nectar-collecting visits. The reason for using data for all castes combined was to provide an overall measure of dietary similarity between species (competition could occur among or between castes).

Since tongue length is known to influence floral preferences, tongue lengths were measured for ten workers of each species, or for as many workers as were captured for the scarcest species (measured as length of the glossa plus prementum, following PrysJones and Corbet (1991)). To compare the diet breadth of the species recorded, a Simpson's index was calculated for the diversity of flowers visited by each caste (Simpson 1949):

$$
D_{s}=\sum_{i=1}^{s} \frac{\left(n_{i}\left(n_{i}-1\right)\right)}{(N(N-1))}
$$

where $n_{i}$ is the number of flowers of the ith plant species that were visited, $\mathrm{N}$ is the total number of flowers visited, and $s$ is the total number of flower species visited. As is 
usual, results are presented as 1/D, so that larger values indicate higher diversity. This index is insensitive to sample size (Magurran 1988) which is important because samples are inevitably larger for the more common species. Only species for which there were at least 10 records of either pollen or nectar collection per caste were included. Note that Simpson's index was also calculated for the diversity of bee species present within sites.

Williams (2005) criticises the use of Simpson's index for measuring diet breadth, arguing that use of rarefaction is preferable to take into account varying sample sizes across bee species. Hence we also calculated diet breadth for each bee species using rarefaction, randomly sub-sampling 10 visits from those recorded, without replacement, and repeating the procedure 100 times. This was carried out separately for workers collecting pollen, workers collecting nectar, and for males. This provides an estimate of the mean number of plant species each bee species would be expected to visit in a total of 10 flower visits.

Niche overlap in flower usage was calculated between species pairs for the seven most abundant bumblebee species following Colwell and Futuyma (1971). A separate measure was calculated for pollen and nectar collection.

Niche overlap between bee species $j$ and $h=1-0.5 \sum_{k}\left(P_{j k}-P_{h k}\right)$

Where $P_{j k}=\frac{\text { No. bee species } j \text { visiting plant species } k}{\text { Total no. bee species } j}$

To examine whether niche differentiation in either nectar or pollen collection could be explained by differences in tongue length between species, the relationship between niche 
overlap and tongue length between each species pair was examined with a Mantel test with 10,000 runs, using MANTEL 2.0 (Manly 1986, Liedloff 1999).

Patterns of community composition were examined as follows. Sites were classified according to altitude, $<600 \mathrm{~m}$ (9 sites), 600-900m (12 sites), >900m (11 sites). These altitudes correspond to: farmed lowland valleys (mostly arable with some pasture); steep hillsides (mostly pasture with limited arable cropping); montane pasture mixed with coniferous forest. For each bee species, the probability of it being present at any randomly chosen site within an altitudinal class was calculated (the proportion of sites in which it was recorded). For each possible pair of species and altitudinal class, the probability of both being present in any one site was then calculated (assuming that they were independently distributed). This probability was then used to predict the number of sites within each altitudinal class in which a pair of species would be expected to co-occur, for comparison (by paired t-test) with the observed number of sites in which both actually occurred. Finally, the expected values were subtracted from the observed values to give an index with negative values indicating that species occurred together less often than expected by chance, and positive values indicating that species co-occurred more frequently than a random distribution would predict. This index was correlated with the niche overlap and difference in tongue length between species pairs using a Mantel test.

\section{RESULTS}

In total 2,001 individual bumblebees belonging to 23 different species were recorded (Figure 1), of which 233 were collecting pollen and the remaining 1,768 were collecting nectar. Both species richness and diversity (Simpson's 1/D) varied significantly with altitude, with richness peaking at about $900 \mathrm{~m}$ and diversity at approximately $1,100 \mathrm{~m}$, an altitude at which the habitat is predominantly small montane hay meadows and pasture 
amongst coniferous forest (Fig 2). Both relationships were best described by quadratic curves (Fig 2., $r^{2}=0.414, F_{2,29}=10.26, p<0.001$ and $r^{2}=0.360, F_{2,29}=8.17, p=0.002$ for richness and diversity, respectively). No bees were observed above $1580 \mathrm{~m}$, and very few flowers were present above this altitude. Some bee species exhibited marked altitudinal preferences; $B$. humilis and $B$. terrestris were predominantly found the lowest sites, while B. pyrenaeus, B. wurflenii and B. pratorum were more abundant at high altitude (in the latter, this is probably because in the lowlands the colony cycle had ended by August) (Table 1). Male cuckoo bumblebees were also mainly recorded at high altitude. Some species were found throughout the altitudinal range studied up to $1580 \mathrm{~m}$, including $B$. hortorum, B. lucorum and B. lapidarius.

By far the most favoured source of pollen was Trifolium pratense, which comprised $51 \%$ of pollen-collecting visits by bumblebees (species and sites combined) (Table 2). More broadly, Fabaceae comprised over $70 \%$ of pollen-collecting visits. Nectar-collection was spread more equitably among plant species and families, with the three most preferred sources being Epilobium angustifolium, T. pratense and Centaurea nigra. (Table 3, see also Appendix 2).

The principal components analysis separated bee species (castes combined) according to their overall forage use (Fig. 3). Component 1 is most strongly (and negatively) correlated with visits to Fabaceae (primarily $T$. pratense), and positively correlated with visits to Dipsacaceae (mainly Knautia arvensis) and Onagraceae (mainly Epilobium angustifolium). Component two is positively correlated with visits to Apiaceae and Lamiacae, and negatively correlated with visits to Ranunculaceae (largely Aconitum spp.). The individual bee species cluster partly according to subgenus, with the two long-tongued Megabombus species on the far left both of which feed predominantly on T. pratense. The medium- 
tongued Thoracobombus species cluster at the centre left, feeding largely on Fabaceae and to a lesser extent Lamiaceae and Boraginaceae (primarily Symphytum officinale). The two short-tongued subgenus Bombus species are at the top right, and are characterised by a polylectic diet including Apiaceae which were never visited by any other bumblebee species. The seven most common species (circled) are well separated in niche space using these two principal components. Notably, PCA 1 is strongly negatively related to tongue length (linear regression, $r^{2}=0.723, F_{1,14}=36.4, p<0.001$ ) (Figure 4).

Diet breadth as measured by Simpson's index was strongly correlated with the measure of diet breadth obtained by rarefaction $\left(r^{2}=0.975, n=6, p<0.001 ; r^{2}=0.745, n=14, p=\right.$ $0.002 ; r^{2}=0.953, n=11, p<0.001$ for pollen collection, worker nectar collection and male nectar collection, respectively). Diet breadth when collecting pollen or nectar as measured by Simpson's index was not significantly correlated with overall abundance of each bee species (Pearson's product-moment correlation coefficient $r=0.307, n=6$, n.s.; $r=0.153$, $n=14$, n.s.; $r=0.425, n=12$, n.s. for pollen collection, worker nectar collection and male nectar collection, respectively). Similarly diet breadth was not correlated with the number of sites at which each bee species was recorded $(r=0.097, n=6$, n.s.; $r=0.181, n=14$, n.s.; $r=0.352, n=12$, n.s. for pollen collection, worker nectar collection and male nectar collection, respectively). In contrast, when these analyses were repeated using diet breadth as calculated by rarefaction, a significant positive relationship emerged between worker diet breadth when collecting nectar and abundance $(r=0.207, n=6$, n.s.; $r=$ 0.579, $n=14, p=0.030 ; r=0.342, n=12$, n.s. for pollen collection, worker nectar collection and male nectar collection, respectively) (Fig 5). There was also a positive relationship between worker diet breadth when collecting nectar and the number of sites in which a bee species was recorded $(r=0.008, n=6$, n.s.; $r=0.631, n=14, p=0.016 ; r=$ 
$0.355, \mathrm{n}=12$, n.s. for pollen collection, worker nectar collection and male nectar collection, respectively) (Fig 5).

Within species, worker diet breadth when collecting pollen as measured by Simpson's index was significantly narrower than when collecting nectar (mean \pm SE: $2.99 \pm 0.50$ and $7.09 \pm 1.19$ for pollen and nectar respectively; paired t-test, $t=3.24$, d.f. $=5, p=0.023$ ) However, this relationship was not significant when using diet breadth calculated by rarefaction (mean \pm SE: $4.71 \pm 0.67$ and $5.62 \pm 0.40$ for pollen and nectar respectively; paired t-test, $t=1.67$, d.f. $=5, p=0.156)$.

Comparing males and workers of the same species, diet breadth when collecting nectar is positively correlated whichever method of calculating diet breadth was used $(r=0.634$, $n=10, p<0.05$ and $r=0.802, n=10, p=0.005$ for Simpson's index and rarefaction, respectively) i.e. in species where the workers have a broad diet the males are also likely to have a broad diet. Diet breadth of males and females when collecting nectar did not differ whichever method of calculating diet breadth was used (paired t-test, $t=0.69$, d.f. $=$ 9 , n.s. and $t=2.05$, d.f. $=9$, n.s. for Simpson's index and rarefaction, respectively). Interestingly, tongue length and diet breadth when collecting pollen were strongly negatively correlated $(r=-0.870, n=6, p=0.024$, Fig 6 , for Simpson's index; $r=-0.932, n$ $=6, p=0.007$ using diet breadth calculated by rarefaction). In contrast, tongue length and diet breadth when collecting nectar were not related for either workers $(r=-0.16, n=14$, n.s. and $r=-0.254, n=14$, n.s. for Simpson's index and diet breadth calculated by rarefaction, respectively) or males $(r=0.23, n=12$, n.s. and $r=0.33, n=12$, n.s. for Simpson's index and rarefaction, respectively). 
There was no relationship between dietary niche overlap of workers when collecting pollen and difference in mean tongue length between species pairs (Mantel test, correlation = $0.272, p=0.164)$, although only six species could be included in this analysis since too few data on pollen collection were available from the majority of species. However, there was a clear negative correlation between dietary niche overlap when collecting nectar and difference in tongue length for both workers (14 species included, Mantel test, correlation $=-0.449, p=0.003)$ and for males $(12$ species included, Mantel test, correlation $=-0.419$, $p=0.025)$

In all three altitudinal classes, bumblebee species exhibited evidence of positive assortment among sites i.e. on average, species co-occurred more frequently than a random distribution would predict, although this was not significant for intermediate altitudes (paired t-tests: $t=3.74$, d.f. $=104 ; p<0.001$ for $<600 m ; t=1.60$, d.f. $=104$; $n . s$. for $600-900 m ; t=3.82$, d.f. $=104 ; p<0.001$ for $>900 m)$. However, the likelihood of a particular species pair co-occurring was not influenced by the degree of niche overlap for workers collecting nectar (Mantel tests, correlation coefficients $-0.119,0.138$ and 0.097 for sites at $<600 \mathrm{~m}, 600-900 \mathrm{~m}$ and $>900 \mathrm{~m}$, respectively, all non significant) or for workers collecting pollen (Mantel tests, correlation coefficients $-0.060,0.045$ and 0.047 , respectively).

Similarly, the likelihood of species co-occurring was not influenced by their relative tongue lengths i.e. species with similar tongue lengths were just as likely to co-occur as species with very different tongue lengths (Mantel tests, correlation coefficients $0.028,0.050$ and 0.041 for sites at $<600 \mathrm{~m}, 600-900 \mathrm{~m}$ and $>900 \mathrm{~m}$, respectively, all non significant).

\section{DISCUSSION}

Both bumblebee richness (23 species) and diversity in southern Poland were higher than described in similar studies in northern Europe (11-15 species per $100 \mathrm{~km}$ square) or 
Salisbury Plain in southern UK (15 species) (Ranta 1982, Goulson and Darvill 2004).

Richness and diversity peaked in mountain meadows at $\sim 900-1,100$ m altitude, areas where the high-altitude specialists such as $B$. pyrenaeus overlapped with lowland species such as $B$. ruderatus and $B$. ruderarius. Several species were at their most abundant at these altitudes, including B. soroeensis and B. hypnorum. Four of the five species with the broadest altitudinal ranges (B. lucorum, B. hortorum, B. lucorum, B. pratorum but not $B$. wurflenii) are among the most widespread and abundant of European bumblebee species, presumably because they have broad climatic tolerances that allow them to thrive at both a range of altitudes and latitudes (Williams 2005). Peak diversity at intermediate altitudes is broadly in accordance with Connell's (1978) intermediate disturbance hypothesis, since the lower altitudes sampled were relatively intensely farmed whilst the highest altitudes were little disturbed by agriculture. This finding also concurs with studies of bumblebee distributions in Kashmir which describe highest diversity at intermediate altitudes (albeit in much higher mountains) (Williams 1991).

In terms of forage use the results of this study strongly concur with similar studies conducted in Europe in that $T$. pratense in particular and Fabaceae in general provide a large proportion of all pollen collected by bumblebees (Prys-Jones 1982, Teräs 1985, Jennersten et al. 1988, Rasmont 1988, Edwards 1998, Carvell 2002, Goulson and Darvill 2004, Goulson and Hanley 2004, Goulson et al. 2005, Goulson et al. 2006). They also concur with Goulson et al. (2005) in that bumblebees vary greatly in their diet breadth when collecting pollen, with some such as $B$. hortorum gathering pollen almost exclusively from $T$. pratense. Others such as $B$. soroeensis are far more polylectic. Using data from the UK, Goulson et al. (2005) used principal components analysis to separate bumblebee species according to diet, and found that the first principal component was strongly correlated with dependence on Fabaceae. This produced a group of Fabaceae specialists, 
including B. hortorum, B. ruderatus, B. humilis, B. pascuorum and B. lapidarius.

Reassuringly, in the present study the first principal component is again strongly correlated with visitation to Fabaceae, and produces a very similar cluster of species. The only notable difference is the inclusion of $B$. sylvarum and exclusion of $B$. lapidarius from this group when comparing Poland with the UK. A further similarity between studies is that both found there to be a strong correlation between principal component 1 and tongue length, the Fabaceae specialists tending to have longer tongues than other species.

Tongue length has been suggested as a major factor determining which species coexist within bumblebee communities, based on studies in Colorado (Pyke 1982). In short, Pyke argues that species with similar length tongues cannot coexist because they compete strongly for floral resources. Goulson and Darvill (2004) and the present study agree in that niche overlap between species is high when they have tongues of similar length. However, previous studies in Europe have failed to find relationships between tongue length and coexistence, and it is common to find several abundant coexisting species with similar length (short) tongues (Ranta and Vepsäläinen 1981, Ranta 1982, 1983, Ranta and Tiainen 1982, Williams 1985b, 1988). Our study concurs, in that we were unable to find any relationships between co-occurrence of species pairs and the similarity of their tongue length or the degree to which their diets overlapped. Sample sites commonly contained a number of coexisting short, medium and long-tongued species. Indeed, species tended to co-occur more often than would be expected from a random distribution, rather than less often as would be predicted if there was competitive exclusion. This may simply because some sites had more floral resources than others, and a site with abundant resources is likely to attract more bees. However, it is notable that the most abundant species in this study occupy markedly different niche space in terms of forage use (i.e. the circled species are widely spaced in Figure 3). Thus sites generally contained one common long-tongued 
species (B. hortorum) and sometimes also B. ruderatus, but the latter always at low density. Most sites also contained the medium-tongued B. pascuorum in abundance and also much smaller numbers of other medium-tongued species such as $B$. sylvarum, $B$. ruderarius and $B$. veteranus. Three short-tongued species commonly co-occurred and were abundant (B. lucorum, B. soroeensis and B. pratorum), but as can be seen from Fig. 3 their diets are dissimilar. B. soroeensis and B. pratorum are closest, but even for this species pair niche overlap is low (niche overlap for workers collecting nectar $27 \%$, no estimate available for pollen). Thus although species with similar length tongues tend to have greater niche overlap, there is still considerable scope for niche differentiation among species with tongues that are near-identical in length. It is interesting to speculate that Bombus communities in Europe and Asia may have achieved higher levels of niche packing through their longer co-evolutionary history (bumblebees probably arrived in Europe 24-33mya) compared to similar communities in the Americas where they are relatively recent colonists (Williams, 1985a). Alternatively, niche differentiation among species with similar tongue lengths in Europe may reflect the greater higher-level taxonomic diversity present in Europe compared to the Americas i.e. species from different subgenera are more likely to differ in both diet and other aspects of their ecology than species from the same subgenus.

It must be noted that the failure to find evidence of competition shaping community structure in this study must not be interpreted as proof that competition is not occurring. Competitive exclusion may not be evident because queen dispersal each spring masks local competitive effects. Competition might occur earlier in the year, for example in spring when floral resources may be scarce. However, if competitive exclusion were leading to the failure of nests of less competitive species in areas where a superior competitor was abundant, we would expect these patterns to remain in August when the colony cycle is at 
its climax. Alternatively, competitive effects may be obscured by the long foraging range of bumblebee species and the confounding effects of the highly heterogenous spatial and temporal distributions of floral resources. Competition is notoriously hard to convincingly demonstrate in wild populations, requiring replicated exclusion experiments, which are near to impossible with mobile organisms such as bumblebees.

Interestingly, the UK and Polish data differ in one important aspect. In the UK, the Fabaceae specialists tended to be rare, while more polylectic species were more abundant (Goulson et al. 2005). This pattern is not evident in the Polish data, although the Fabaceae specialists do include a number of the less abundant species (B. humilis, $B$. ruderarius, $B$. veteranus, B. ruderatus). Goulson et al. (2005) interpret the rarity of Fabaceae specialists in the UK as the result of the wholesale loss of Fabaceae-rich unimproved grasslands (Howard et al. 2003). It seems probable that the reason many of these species remain moderately abundant in southern Poland is that unimproved pasture remains common for the moment. However, inclusion of Poland and other central and eastern European countries within the European Union may lead to agricultural intensification in the near future, which would threaten currently high levels of biodiversity.

The parallels between studies in the UK and Poland are striking, and provide reassurance that these are general patterns. However, it must be noted that the species composition of both studies overlap heavily, so the studies are not truly independent. It would be exceedingly interesting to carry out similar studies in regions where the bumblebee community is quite different (e.g. America, China, Japan).

\section{ACKNOWLEDGEMENTS}


This work was funded by a grant from the British Ecological Society. Ben Darvill is funded by the Leverhulme Trust, and Gillian Lye by a NERC studentship. Thanks are due to Dr Paul Williams for comments on the MS.

\section{REFERENCES}

Barrow DA, Pickard RS (1984) Size-related selection of food plants by bumblebees. Ecological Entomology 9:369-373

Brian AD (1954) The foraging of bumble bees Part 1. Foraging behaviour. Bee World 35:61-67

Brian AD (1957) Differences in the flowers visited by four species of bumble-bees and their causes. Journal of Animal Ecology 21:223-240

Buchmann SL, Nabhan GP (1996) The Forgotten Pollinators. Island Press, Washington (DC).

Carvell C (2002) Habitat use and conservation of bumblebees (Bombus spp.) under different grassland management regimes. Biological Conservation 103:33-49

Colwell RK, Futuyma DJ (1971) On the measurement of niche breadth and overlap, Ecology 52:567-576

Connell JH (1978) Diversity in tropical rain forests and coral reefs - high diversity of trees and corals is maintained only in a non-equilibrium state. Science 199:1302-1310

Edwards M (1998) U.K. Biodiversity Action Plan Bumblebee Working Group Report 1998. Unpublished report for the UK BAP bumblebee working group, Midhurst, UK.

Fitzpatrick U, Murray TE, Paxton RJ, Breen J, Cotton D, Santorum V, Brown MJF (2007) Rarity and decline in bumblebees - A test of causes and correlates in the Irish fauna. Biological Conservation 136:185-194

Goulson D (2003a) Bumblebees; their behaviour and ecology. Oxford University Press, Oxford. 234pp

Goulson D (2003b) Conserving wild bees for crop pollination. International Journal of Food, Agriculture and the Environment 1:142-144 
Goulson D, Darvill B (2004) Niche overlap and diet breadth in bumblebees; are rare species more specialized in their choice of flowers? Apidologie 35:55-64

Goulson D, Hanley ME (2004) Distribution and forage use of exotic bumblebees in South Island, New Zealand. New Zealand Journal of Ecology 28:225-232

Goulson D, Hanley ME, Darvill B, Ellis JS (2006) Biotope associations and the decline of bumblebees (Bombus spp.). Journal of Insect Conservation 10:95-103

Goulson D, Hanley ME, Darvill B, Ellis JS, Knight ME (2005) Causes of rarity in bumblebees. Biological Conservation 122:1-8

Goulson D, Hughes WOH, Derwent LC, Stout JC (2002b) Colony growth of the bumblebee, Bombus terrestris, in improved and conventional agricultural and suburban habitats. Oecologia 130:267-273

Goulson D, Peat J, Stout JC, Tucker J, Darvill B, Derwent LC, Hughes WOH (2002a) Can alloethism in workers of the bumblebee Bombus terrestris be explained in terms of foraging efficiency? Animal Behavaviour 64:123-130

Goulson D, Stout JC, Hawson SA, Allen JA (1998) Floral display size in comfrey, Symphytum officinale L. (Boraginaceae); relationships with visitation by three bumblebee species and subsequent seed set. Oecologia 113:502-508

Graham L, Jones KN (1996) Resource partitioning and per-flower foraging efficiency in 2 bumble bee species. American Midlands Naturalist 136:401-406

Harder LD (1985) Morphology as a predictor of flower choice by bumblebees. Ecology 66:198-210

Heinrich B (1976) Resource partitioning among some eusocial insects: Bumblebees. Ecology 57:874-889

Hobbs GA, Nummi WO, Virostek JF (1961) Food-gathering behaviour of honey, bumble, and leaf-cutter bees (Hymenoptera: Apoidea) in Alberta. Canadian Entomologist 93:409-419

Hobbs GA, Nummi WO, Virostek JF (1962) Managing colonies of bumble bees (Hymenoptera: Apidae) for pollination purposes. Canadian Entomologist 94:11211132 
Holm SN (1966) The utilization and management of bumblebees for red clover and alfalfa seed production. Annual Review of Entomology 11:155-182

Howard DC, Watkins JW, Clarke RT, Barnett CL, Stark GJ (2003) Estimating the extent and change in broad habitats in Great Britain. Journal of Environmental Management 67:219-227

Inouye DW (1978) Resource partitioning in bumblebees: experimental studies of foraging behavior. Ecology 59:672-678

Inouye DW (1980) The effects of proboscis and corolla tube lengths on patterns and rates of flower visitation by bumblebees. Oecologia 45:197-201

Jennersten O, Berg L, Lehman C (1988) Phenological differences in pollinator visitation, pollen deposition and seed set in the sticky catchfly Viscaria vulgaris. Journal of Ecology 76:1111-1132

Johnson RA (1986) Intraspecific resource partitioning in the bumble bees Bombus ternarius and B. pensylvanicus. Ecology 67:133-138

Kells AR, Holland J, Goulson D (2001) The value of uncropped field margins for foraging bumblebees. Journal of Insect Conservation 5:283-291

Kosior A (1995) Changes in the fauna of bumble-bees (Bombus Latr.) and cuckoo-bees (Psithyrus Lep.) of selected regions in southern Poland. In: Banaszak J (Ed.), Changes in Fauna of Wild Bees in Europe, Bydgoszcz, Pedagogical University, pp103-111

Kosior A, Celary W, Olejnikzak P, Fijal J, Krol W, Solarz W, Plonka P (2007) The decline of the bumble bees and cuckoo bees (Hymenoptera: Apidae: Bombini) of Western and Central Europe. Oryx 41:79-88

Liedloff A (1999) Mantel nonparametric test calculator (version 2.0). Available from http://www.sci.qut.edu.au/NRS/Mantel.htm

Magurran AE (1988) Ecological diversity and its management, Princeton University Press, Princeton 
Manly BFJ (1986) Randomization and regression methods for testing for associations with geographical, environmental and biological distances between populations.

Researches on Population Ecology 28:201-218

Osborne JL, Corbet SA (1994) Managing habitats for pollinators in farmland. Aspects of Applied Biology 40:207-215

Peat J, Goulson D (2005a) Effects of experience and weather on foraging efficiency and pollen versus nectar collection in the bumblebee, Bombus terrestris. Behavioural Ecology and Sociobiology 58:152-156

Peat J, Tucker J, Goulson D (2005b) Does intraspecific size variation in bumblebees allow colonies to efficiently exploit diverse floral resources? Ecological Entomology 30, $176-181$

Prys-Jones OE (1982) Ecological studies of foraging and life history in bumblebees. Ph.D. thesis, University of Cambridge

Prys-Jones OE, Corbet SA (1991) Bumblebees. Richmond Publishing Company, Slough Pyke GH (1982) Local geographic distributions of bumblebees near Crested Butte, Colorado: competition and community structure. Ecology 63:555-573

Ranta E (1982) Species structure of North European bumblebee communities. Oikos $38: 202-209$

Ranta E (1983) Proboscis length and the coexistence of bumblebee species. Oikos 43:189-196

Ranta E, Lundberg H (1980) Resource partitioning in bumblebees: the significance of differences in proboscis length. Oikos 35:298-302

Ranta E, Lundberg H, Teräs I (1981) Patterns of resource utilization in two Fennoscandian bumblebee communities. Oikos 36:1-11

Ranta E, Tiainen M (1982) Structure in seven bumblebee communities in eastern Finland in relation to resource availability. Holarctic Ecology 5:48-54

Ranta E, Vepsäläinen K (1981) Why are there so many species? Spatio-temporal heterogeneity and northern bumblebee communities. Oikos 36:28-34 
Rasmont $P$ (1988) Monographie écologique et zoogéographique des bourdons de France et de Belgique (Hymenoptera, Apidae, Bombinae). Ph.D. thesis, Faculté des Sciences Agronomique de l'Etat, Gembloux, Belgium.

Rasmont $P$ (1995) How to restore the Apoid diversity in Belgium and France? Wrong and right ways, or the end of protection paradigm! In: Banaszak J (Ed.), Changes in Fauna of Wild Bees in Europe, Bydgoszcz, Pedagogical University, pp53-64 Simpson GH (1949) Measurement of diversity. Nature 163:688 Tepedino VJ, Stanton NL (1981) Diversity and competition in bee-plant communities on short-grass prairie. Oikos 36:35-44

Teräs I (1976) Flower visits of bumblebees, Bombus Latr. (Hymenoptera: Apidae) during one summer. Annales Zoologici Fennici 13:200-232

Teräs I (1985) Food plants and flower visits of bumble-bees (Bombus: Hymenoptera, Apidae) in southern Finland. Acta Zoologica Fennica 179:1-120

Westrich P (1996) Habitat requirements of central European bees and the problems of partial habitats. In: Matheson A, Buchmann SL, O'Toole C, Westrich P, Williams IH (Eds), The conservation of bees, Academic Press, London, pp. 2-16

Westrich P, Schwenninger H-R, Dathe H, Riemann H, Saure C, Voith J, Weber K (1998)

Rote Liste der Bienen (Hymenoptera: Apidae). In: Rote Liste Gefährdeter Tiere

Deutschlands. Ed. By Bundesamt für Naturschutz. Naturschutz 55, Bonn, Schriftenr. Landschaftspf, pp. 119-129

Williams PH (1982) The distribution and decline of British bumble bees (Bombus Latr). Journal of Apicultural Research 21:236-245

Williams PH (1985a) A preliminary cladistic investigation of relationships among the bumble bees (Hymenoptera, Apidae). Systematic Entomology 10:239-255 Williams PH (1985b) On the distribution of bumble bees (Hymenoptera, Apidae), with particular regard to patterns within the British Isles. PhD thesis, University of Cambridge, UK. 180 pp. 
Williams PH (1986) Environmental change and the distribution of British bumble bees (Bombus Latr.). Bee World 67:50-61

Williams PH (1988) Habitat use by bumble bees (Bombus spp.). Ecological Entomology $13: 223-237$

Williams PH (1989) Why are there so many species of bumble bees at Dungeness? Botanical Journal of the Linnean Society 101:31-44.

Williams PH (1991) The bumble bees of the Kashmir Himalaya (Hymenoptera: Apidae, Bombini). Bulletin of the British Museum (Natural History) (Entomology) 60:1-204 Williams PH (2005) Does specialization explain rarity and decline among British bumblebees? A response to Goulson et al.. Biological Conservation 122:33-43 Williams PH Araujo MB, Rasmont P (2007) Can vulnerability among British bumblebee (Bombus) species be explained by niche position and breadth? Biological Conservation 138:493-505 
Table 1. Abundance and characteristics of Bombus species present in this study. Where possible, tongue lengths were measured for ten workers of each species (length of the glossa plus prementum, following Prys-Jones and Corbet (1991)). Museum specimens were used to supplement sample sizes. For B. quadricolor, only one specimen was available in total. Diet breadth is calculated as Simpson's 1/D, for pollen collecting workers, nectar-collecting workers and nectar-collecting males, and also using a rarefaction procedure (values in brackets). Diet breadth is only calculated where a sample size of $>10$ was obtained.

\begin{tabular}{|c|c|c|c|c|c|c|c|c|}
\hline Species & $\begin{array}{l}\text { No sites } \\
\text { present }\end{array}$ & N & $\begin{array}{l}\text { Altitudinal } \\
\text { range }\end{array}$ & $\begin{array}{l}\text { Alt. mean (of } \\
\text { sites } \\
\text { occupied) }\end{array}$ & $\begin{array}{l}\text { Tongue } \\
\text { length } \\
(\mathrm{mm})\end{array}$ & $\begin{array}{l}\text { Diet } \\
\text { breadth } \\
\text { - pollen }\end{array}$ & $\begin{array}{c}\text { Diet } \\
\text { breadth } q \\
\text { - nectar }\end{array}$ & $\begin{array}{c}\text { Diet } \\
\text { breadth } \\
\text { - nectar }\end{array}$ \\
\hline bohemicus & & 8 & $650-970$ & 782 & $7.0 \pm 0.4$ & & & \\
\hline campestris & 8 & 22 & $450-1130$ & 692 & $6.9 \pm 0.4$ & & & $\begin{array}{c}4.77 \\
(5.11)\end{array}$ \\
\hline distinguendus & 1 & 1 & 370 & 370 & $11.0 \pm 0.6$ & & & \\
\hline hortorum & 26 & 185 & $370-1580$ & 825 & $12.7 \pm 0.7$ & $\begin{array}{c}1.15 \\
(1.88)\end{array}$ & $\begin{array}{c}4.10 \\
(4.66) \\
3.30\end{array}$ & $\begin{array}{c}3.82 \\
(5.12)\end{array}$ \\
\hline humilis & 6 & 15 & $370-630$ & 554 & $7.9 \pm 0.4$ & & $\begin{array}{l}(3.66) \\
3.00\end{array}$ & 2.53 \\
\hline hypnorum & 12 & 45 & $400-1130$ & 779 & $6.4 \pm 0.5$ & & $(3.91)$ & $(4.36)$ \\
\hline lapidarius & 26 & 176 & $370-1580$ & 820 & $\begin{array}{l}4.8 \pm 0.3 \\
7.0 \pm 0.4\end{array}$ & $\begin{array}{l}4.00 \\
(6.23)\end{array}$ & $\begin{array}{l}10.5 \\
(7.07)\end{array}$ & \\
\hline lucorum & 28 & $\begin{array}{c}254 \\
1\end{array}$ & $\begin{array}{c}370-1580 \\
970\end{array}$ & $\begin{array}{l}820 \\
970\end{array}$ & $6.1 \pm 0.5$ & $\begin{array}{c}3.31 \\
(5.24)\end{array}$ & $\begin{array}{l}5.74 \\
(5.71)\end{array}$ & $\begin{array}{c}6.61 \\
(6.09)\end{array}$ \\
\hline pascuorum & 28 & 549 & $370-1130$ & 679 & $8.4 \pm 0.6$ & $\begin{array}{c}3.01 \\
(4.66)\end{array}$ & $\begin{array}{c}7.24 \\
(6.47)\end{array}$ & $\begin{array}{c}14.2 \\
(7.55)\end{array}$ \\
\hline pratorum & 21 & 109 & $400-1580$ & 981 & $5.8 \pm 0.3$ & & $(3.94)$ & $\begin{array}{c}(2.91) \\
1.71\end{array}$ \\
\hline pyrenaeus & 8 & 54 & $786-1580$ & 1157 & $6.1 \pm 0.4$ & & & $(3.02)$ \\
\hline quadricolor & 1 & 8 & 1130 & 1130 & 6.6 & & & \\
\hline ruderarius & 14 & 37 & $370-1000$ & 786 & $8.4 \pm 0.5$ & & $\begin{array}{c}3.75 \\
(4.71) \\
3.50\end{array}$ & $\begin{array}{c}6.84 \\
(5.77)\end{array}$ \\
\hline ruderatus & 9 & 18 & $400-970$ & 766 & $11.9 \pm 0.6$ & & (3.64) & \\
\hline rupestris & 2 & 4 & $550-1130$ & 985 & $7.0 \pm 0.4$ & & & \\
\hline $\begin{array}{c}\text { soroeensis } \\
\text { rylvarum }\end{array}$ & 20 & 310 & $450-1400$ & 858 & $6.2 \pm 0.5$ & $\begin{array}{c}4.44 \\
(6.22) \\
2.05 \\
(4.00)\end{array}$ & $\begin{array}{c}4.32 \\
(4.98) \\
10.7 \\
(487)\end{array}$ & $\begin{array}{c}3.58 \\
(4.49) \\
11.3 \\
(7.00)\end{array}$ \\
\hline sylvestris & 2 & 6 & $848-1100$ & 1058 & $6.6 \pm 0.4$ & & & \\
\hline terrestris & 10 & 29 & $400-895$ & 599 & $6.3 \pm 0.5$ & & $\begin{array}{c}9.75 \\
(5.64) \\
5.25\end{array}$ & $7.5(6.05)$ \\
\hline veteranus & 6 & 27 & $754-970$ & 837 & $8.3 \pm 0.5$ & & $\begin{array}{c}(4.75) \\
6.03\end{array}$ & 10.6 \\
\hline wurflenii & 23 & 74 & $400-1580$ & 868 & $7.5 \pm 0.6$ & & $(5.44)$ & $(6.90)$ \\
\hline
\end{tabular}


Community composition in bumblebees 
Table 2. Percentage of all pollen-collecting visits by bumblebees (species combined) according to plant species $(\mathrm{N}=233)$

\begin{tabular}{lccc}
\multicolumn{1}{c}{ Species } & $\%$ & Cumulative $\%$ & Family \\
Trifolium pratense & 51.07 & 51.07 & Fabaceae \\
Hypericum perforatum & 5.58 & 56.65 & Guttiferae \\
Centaurea nigra & 5.58 & 62.23 & Asteraceae \\
Trifolium repens & 5.15 & 67.38 & Fabaceae \\
Symphytum officinale & 5.15 & 72.53 & Boraginaceae \\
Trifolium medium & 4.29 & 76.82 & Fabaceae \\
Lathyrus pratensis & 3.86 & 80.69 & Fabaceae \\
Galeopsis tetrahit & 3.00 & 83.69 & Lamiaceae \\
Lathyrus tuberosus & 3.00 & 86.70 & Fabaceae \\
Vicia cracca & 2.58 & 89.27 & Fabaceae \\
Rhinanthus minor & 2.15 & 91.42 & Scrophulariaceae \\
Epilobium angustifolium & 1.72 & 93.13 & Onagraceae \\
Rubus fruticosus agg. & 1.72 & 94.85 & Rosaceae \\
Lotus corniculatus & 0.86 & 95.71 & Fabaceae \\
Raphanus raphanistrum & 0.43 & 96.14 & Brassicaceae
\end{tabular}


Table 3. Percentage of all nectar-collecting visits by bumblebees (species and castes combined) according to plant species $(\mathrm{N}=1,768)$.

\begin{tabular}{lcrc}
\multicolumn{1}{c}{ Species } & $\%$ & Cumulative \% & Family \\
Epilobium angustifolium & 19.60114 & 19.60 & Onagraceae \\
Trifolium pratense & 15.66952 & 35.27 & Fabaceae \\
Centaurea nigra & 14.98575 & 50.26 & Asteraceae \\
Impatiens glandulifera & 5.299145 & 55.56 & Balsaminaceae \\
Trifolium repens & 3.304843 & 58.86 & Fabaceae \\
Galeopsis tetrahit & 3.133903 & 61.99 & Lamiaceae \\
Knautia arvensis & 3.019943 & 65.01 & Dipsacaceae \\
Mentha spicata & 2.905983 & 67.92 & Lamiaceae \\
Symphytum officinale & 2.735043 & 70.66 & Boraginaceae \\
Carduus crispus & 2.735043 & 73.39 & Asteraceae \\
Cirsium arvense & 2.507123 & 75.90 & Asteraceae \\
Trifolium medium & 2.279202 & 78.18 & Fabaceae \\
Stachys sylvatica & 1.937322 & 80.11 & Lamiaceae \\
Cirsium palustre & 1.823362 & 81.94 & Asteraceae \\
Origanum vulgare & 1.652422 & 83.59 & Lamiaceae
\end{tabular}




\section{Figure Legends}

Figure 1. Total numbers of each bee species (all castes combined) recorded at 32 sites in S. Poland. Subgenus Psithyrus (cuckoo bumblebees) indicated by white bars.

Figure 2. Relationships between bumblebee species richness, Simpson's diversity index $(1 / D)$ and altitude. Lines of best fit: richness $=0.016$ (altitude) $-8.9 \times 10^{-6}(\text { altitude })^{2}+2.80$, $r^{2}=0.414, p<0.001 ; 1 / D=0.015$ (altitude) $-6.9 \times 10^{-6}$ (altitude $)^{2}-2.10, r^{2}=0.360, p=0.002$.

Figure 3. The proportion of visits to different plant families by each bumblebee species (nectar and pollen-collecting visits combined) was subjected to principal components analysis, and the first two components are plotted here. The first and second components account for $25.1 \%$ and $17.4 \%$ of variation in forage use, respectively. Component 1 is strongly negatively correlated with visits to Fabaceae, and positively correlated with visits to Dipsacaceae and Onagraceae. Component two is positively correlated with visits to Apiaceae and Lamiacae, and negatively correlated with visits to Ranunculaceae (Aconitum spp.). The seven most abundant species are circled. Cam = campestris, hort $=$ hortorum, hum $=$ humilis, hyp = hypnorum, lap = lapidarius, luc $=$ lucorum, pasc $=$ pascuorum, pra $=$ pratorum, pyr = pyrenaeus, rrar = ruderarius, rrat = ruderatus, sor = soroeensis, syl = sylvarum, ter $=$ terrestris, vet $=$ veteranus, wur $=$ wurflenii. .

Figure 4. Relationship between tongue length and PCA 1. Key to species names in legend to Fig. 3. 
Figure 5. Relationships between diet breadth of nectar-collecting workers as measured by rarefaction and: a) overall abundance of each bee species; b) number of sites at which each bee species was recorded

Figure 6. Relationship between worker tongue length and diet breadth when collecting pollen as calculated using Simpson's index. This relationship remains when rarefaction is used to calculate diet breadth.. 
Figure 1.

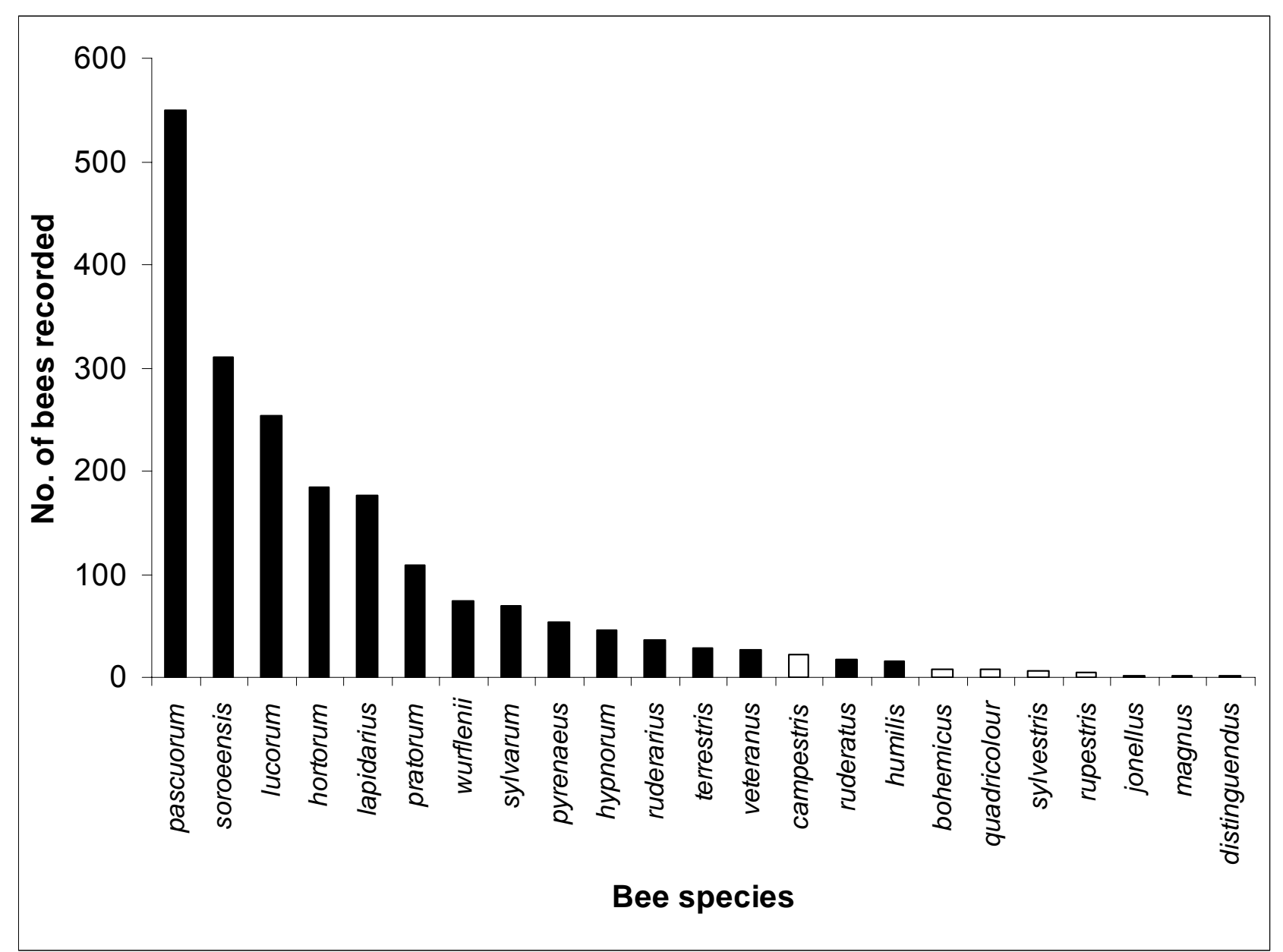


Figure 2.

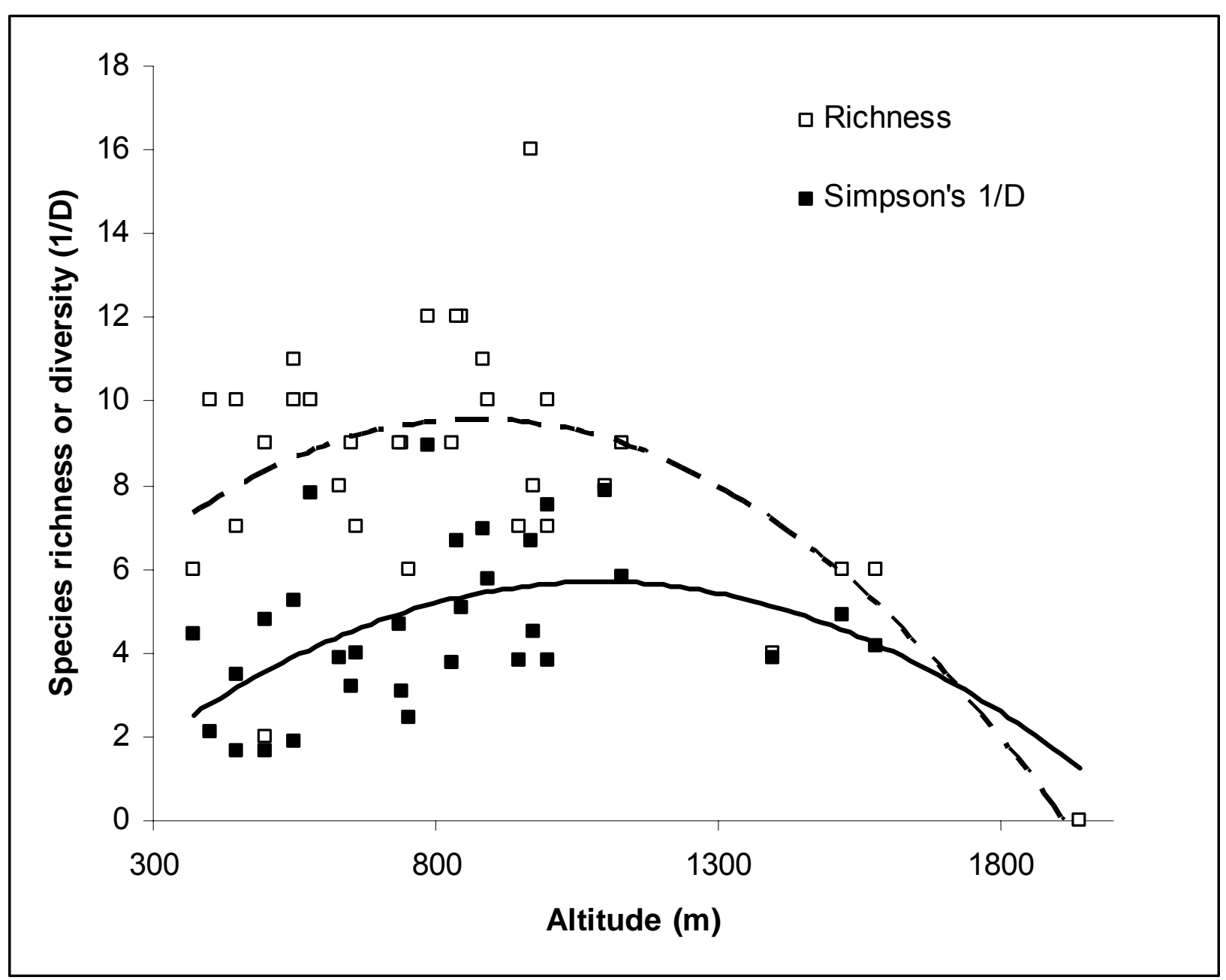


Figure 3.

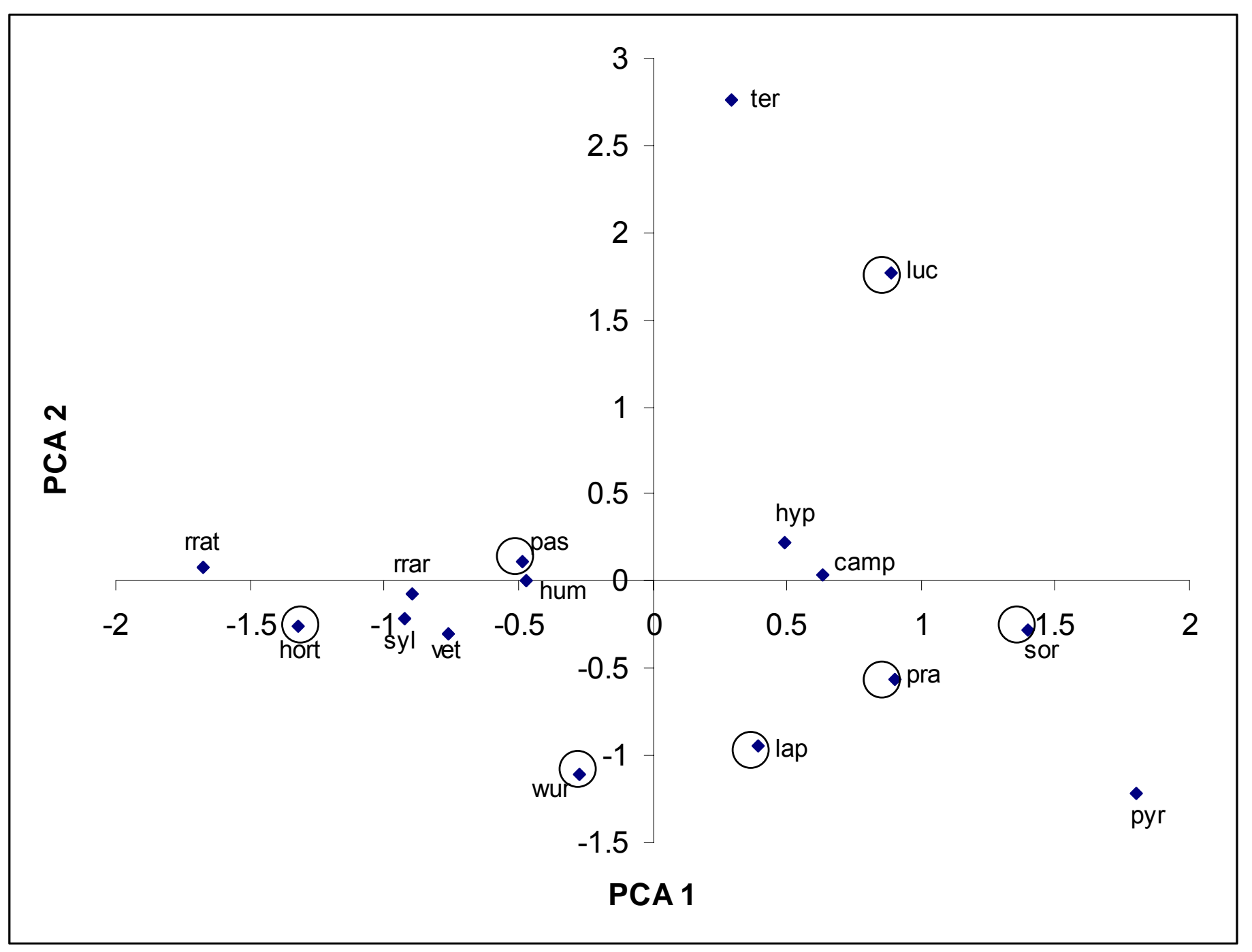


Figure 4.

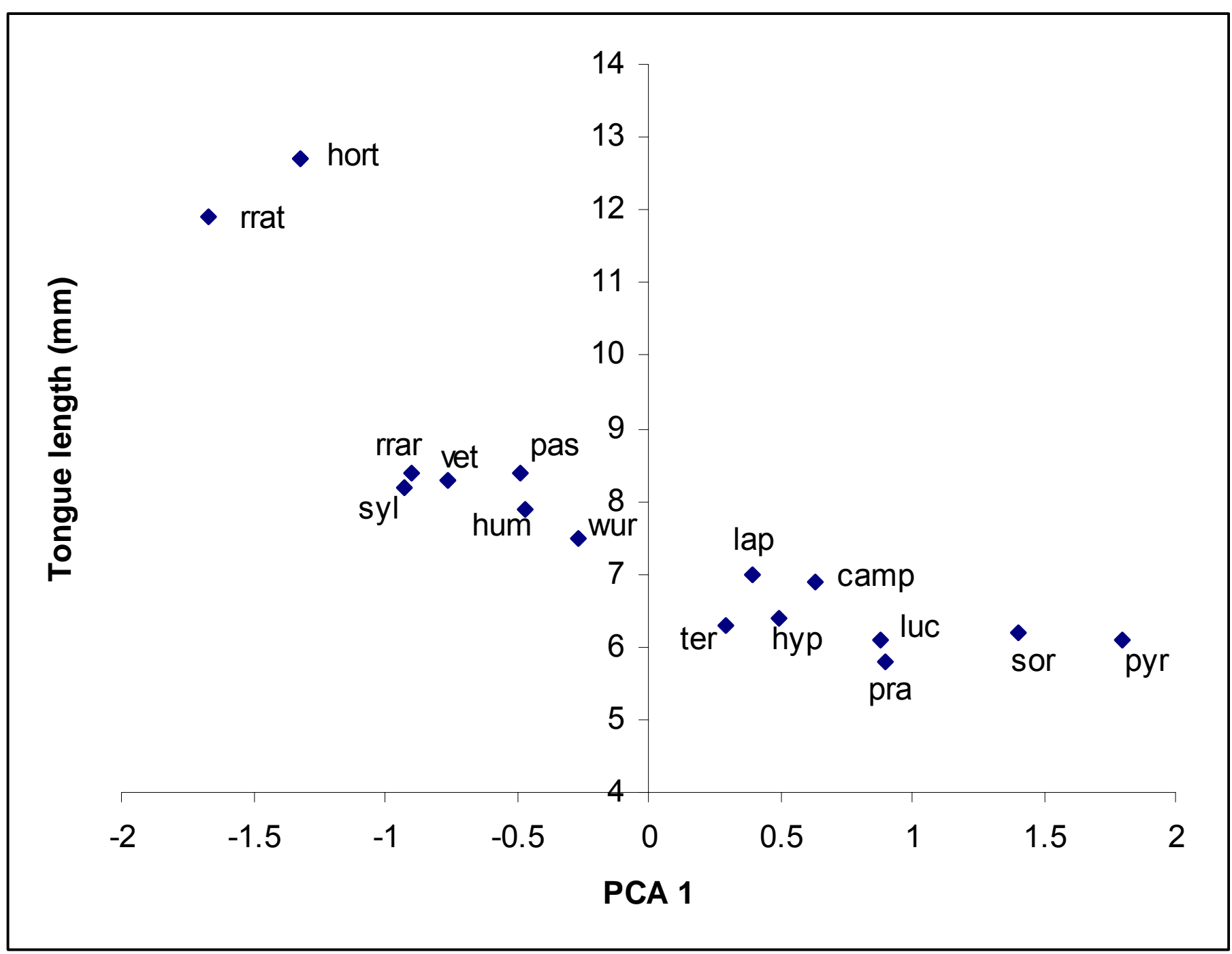


Figure 5.

a)

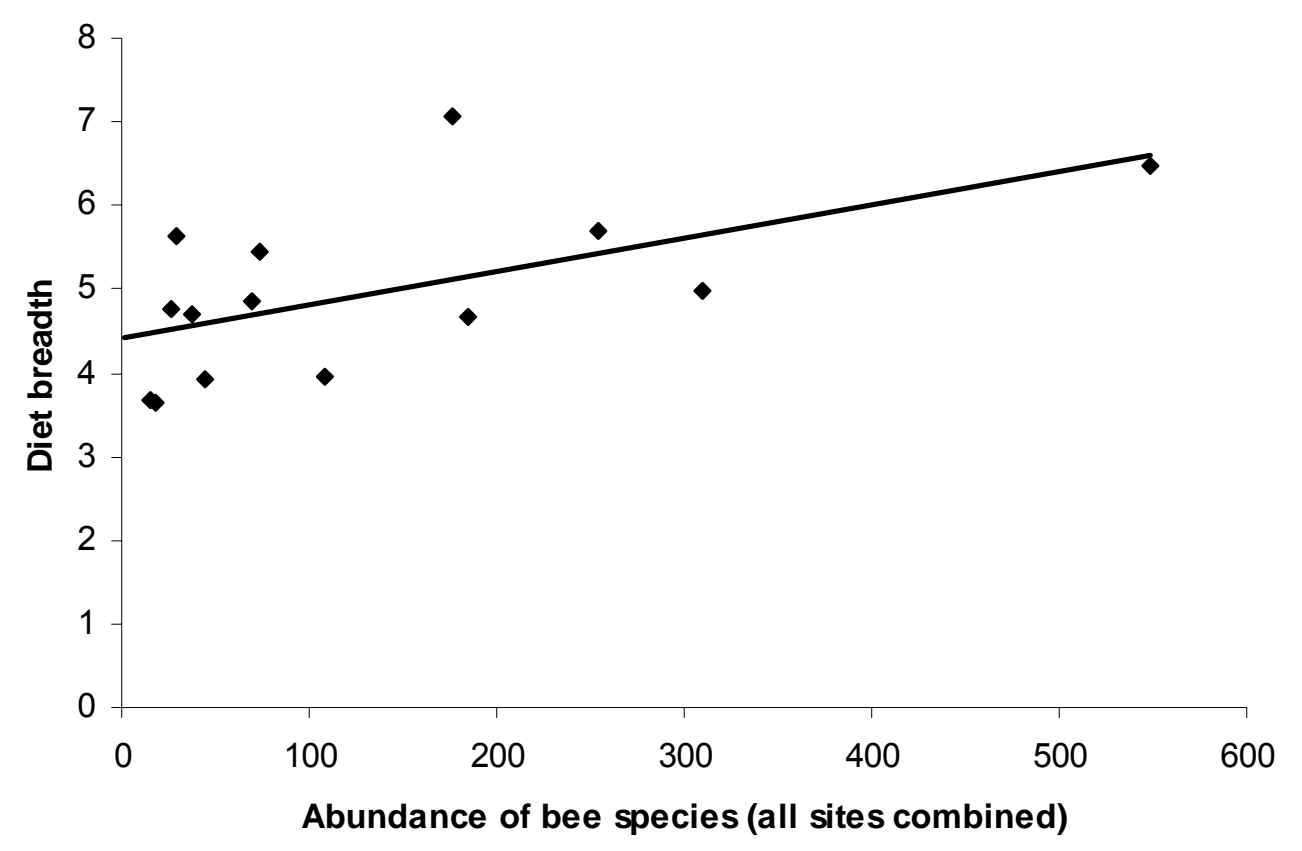

b)

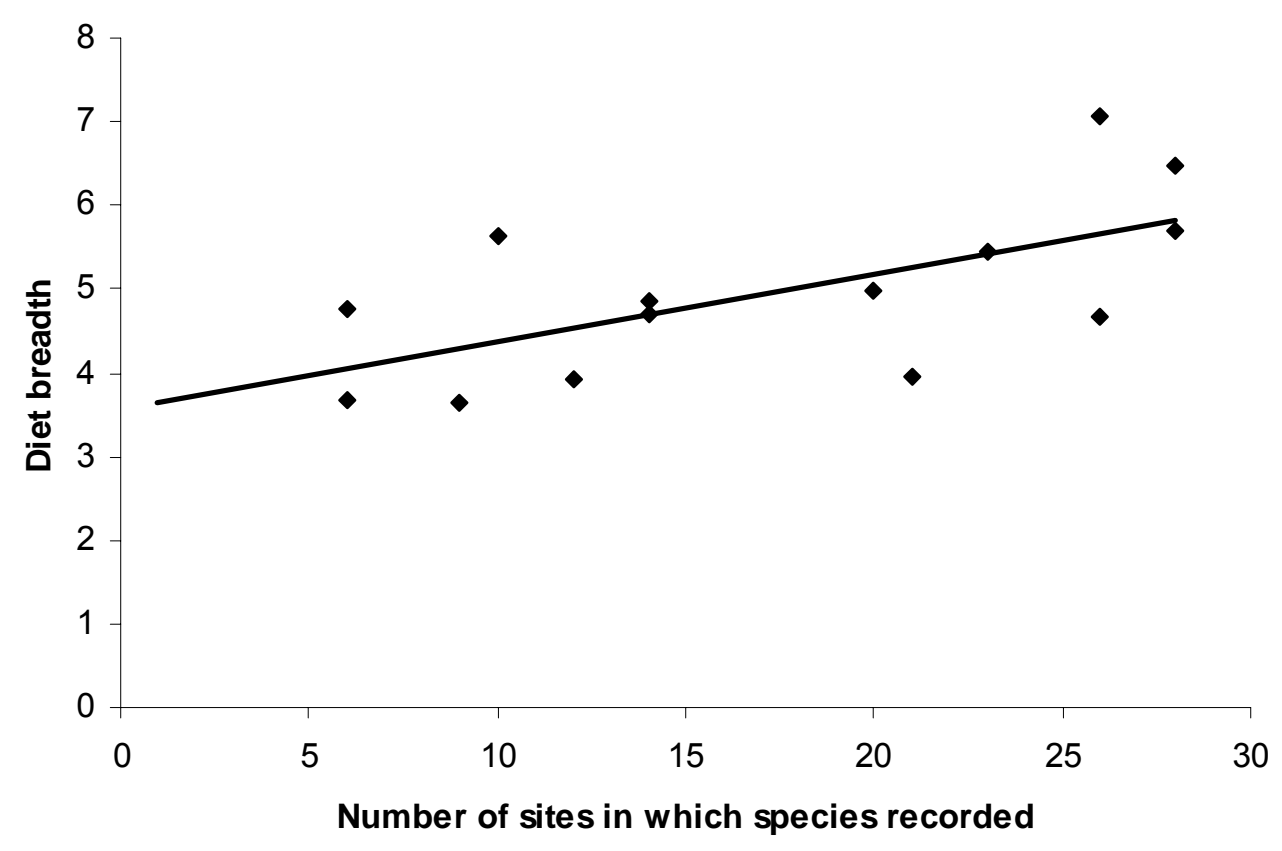


Figure 6.

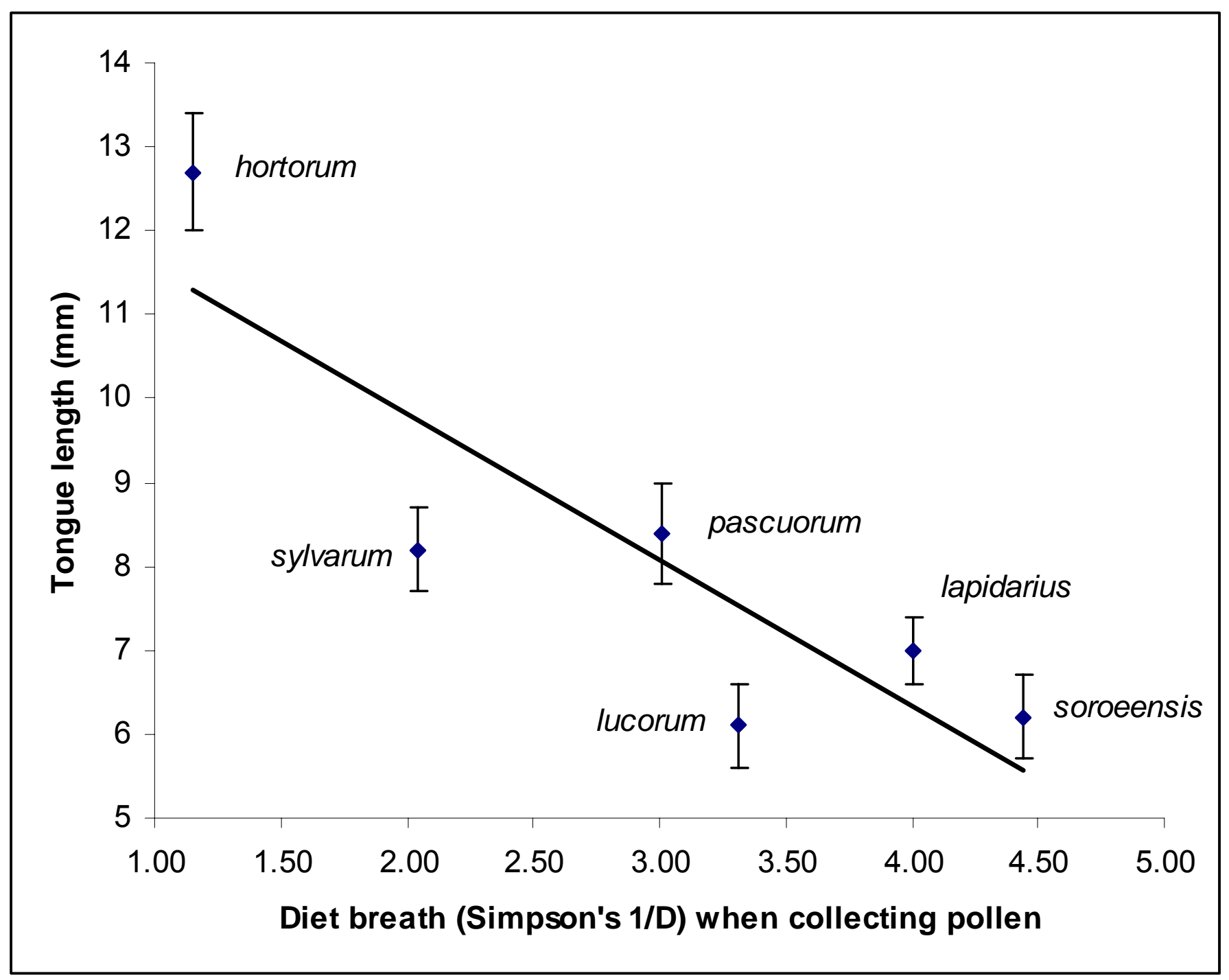

